Journal of Computer Science 7 (11): 1674-1678, 2011

ISSN 1549-3636

(C) 2011 Science Publications

\title{
Traffic Flow Analysis Model based Routing Protocol For Multi-Protocol Label Switching Network
}

\author{
${ }^{1}$ E.R. Naganathan, ${ }^{2}$ S. Rajagopalan and ${ }^{3}$ P. Herbert Raj \\ ${ }^{1}$ Department of Computer Applications, \\ Velammal Engineering College, Chennai, India \\ ${ }^{2}$ Department of CSE, System Engineer, \\ Alagappa University, Karaikudi, India \\ ${ }^{3}$ Department of Technical Education, Brunei
}

\begin{abstract}
Problem statement: Multi-Protocol Label Switching (MPLS) is a mechanism which is used in high-performance telecommunications networks that directs and carries data from one network node to the next with the help of labels. Traffic management is still an issue in MPLS network as it involves high speed internet. Approach: This study proposed a traffic flow analysis of the real time MPLS traffic and segregates the MPLS traffic as three major class based on the outcome of traffic flow analysis. Using the traffic class. This study proposed a reliable transmission methodology which provides traffic free routing in the MPLS networks. Results: The proposed traffic flow analysis based reliable routing model overcomes the network traffic and provides effective routing by offering traffic free path. Conclusion: The proposed traffic flow analysis model outperforms existing routing protocol and offers comparatively negligible packet loss.
\end{abstract}

Key words: Congestion management, traffic flow analysis, proposed traffic, analysis model, routing protocol, routing model, traffic engineering

\section{INTRODUCTION}

MPLS is a highly scalable, protocol agnostic and data-carrying mechanism. Traffic engineering is the major research area in MPLS due to the emerging requirements of MPLS and the internet usage. MPLS operates at an OSI Model layer that is generally considered to lie between traditional definitions of Layer 2 and Layer 3 and thus is often referred to as a "Layer 2.5" protocol, which is shown in the Fig. 1. It was designed to provide a unified data-carrying service for both circuit-based clients and packetswitching clients which provide a datagram service model. It can be used to carry many different kinds of traffic, including IP packets, as well as native ATM, SONET and Ethernet frames. MPLS is currently replacing some of these technologies in the marketplace. It is highly possible that MPLS will completely replace these technologies in the future, thus aligning these technologies with current and future technology needs.
MPLS was originally proposed by a group of engineers from IPSILON Networks, but their "IP Switching" technology, which was defined only to work over ATM, did not achieve market dominance. Cisco Systems, Inc., introduced a related proposal, not restricted to ATM transmission, called "Tag Switching". It was a Cisco proprietary proposal and was renamed "Label Switching". It was handed over to the IETF for open standardization. The IETF work involved proposals from other vendors and development of a consensus protocol that combined features from several vendors' work.

This ensures end-to-end circuits over ANY type of transport medium using ANY network layer protocol. In view of the fact that MPLS supports Internet Protocol revised versions (IPv4 and IPv6), IPX, AppleTalk at Layer3; Ethernet, Token Ring, Fiber Distributed Data Interface (FDDI), Asynchronous transfer Mode (ATM), Frame Relay and PPP (Point to Point Protocol) at Layer 2, it is referred as 'Layer 2.5 protocol'. 


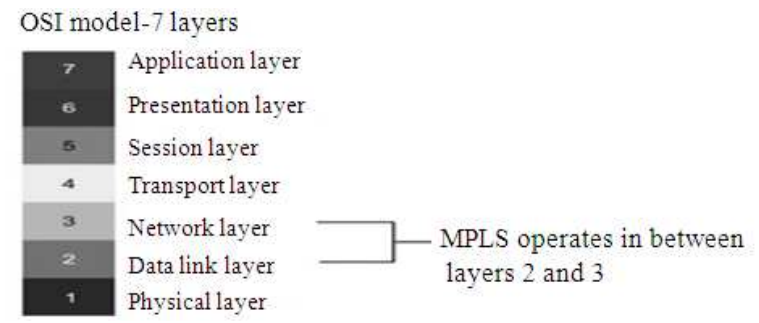

Fig. 1: Operation of MPLS in OSI layer

MPLS works by prefixing packets with an MPLS header, containing one or more "labels". This is called a label stack. Each label stack entry contains four fields:

- A 20-bit label value

- A 3-bit Traffic Class field for QoS (quality of service) priority (experimental) and ECN (Explicit Congestion Notification)

- A 1-bit bottom of stack flag. If this is set, it signifies that the current label is the last in the stack

- An 8-bit TTL (time to live) field

These MPLS-labeled packets are switched after a label lookup/switch instead of a lookup into the IP table. As mentioned above, when MPLS was conceived, label lookup and label switching were faster than a routing table or Routing Information Base (RIB) lookup because they could take place directly within the switched fabric and not the CPU. The entry and exit points of an MPLS network are called Label Edge Routers (LER), which, respectively, push an MPLS label onto an incoming packet and pop it off the outgoing packet. Routers that perform routing based only on the label are called Label Switch Routers (LSR). In some applications, the packet presented to the LER already may have a label, so that the new LER pushes a second label onto the packet.

Traffic engineering is a method of optimizing the performance of a telecommunications network by dynamically analyzing, predicting and regulating the behavior of data transmitted over that network. Traffic engineering is also known as tele-traffic engineering and traffic management. The techniques of traffic engineering can be applied to networks of all kinds, including the Public Switched Telephone Network (PSTN), Local Area Networks (LANs), Wide Area Networks (WANs), cellular telephone networks, proprietary business and the Internet.

The theory of traffic engineering was originally conceived by methods of signal traffic measurement in the early 1900s. A major objective of traffic engineering is to minimize or eliminate high-loss situations. Another goal of traffic engineering is to balance the Quality of Service (QoS) against the cost of operating and maintaining the network.

From the network technology perspective, the integration of the optical layer with electronic layers within a converged data-optical infrastructure, based on classical IP or modern GMPLS (Generalized MPLS) architectures, is a key element in the current trend in broadband network evolution. Two-layer network design problems, where also link and node dimensioning is included in the model, have been considered only quite recently. Some works specifically consider MPLS technology and some of them address the problem of MPLS node location. Given the complexity of the optimization models, several authors rely on path formulations and column generation coupled with branch and bound, joint column and row generation methods, branch and cut with cut-set inequalities or LP-based decomposition approaches. For mid-to-large networks, the solution of choice remains heuristic algorithms, which provide a feasible solution in limited time. However, to the best of our knowledge, the effect of statistical multiplexing has not been previously considered in such network design and routing models.

A virtual private network architecture (Kakooza $e t$ al., 2005) using MPLS, which allows granularity and load balancing. This study shows feasible result in the view of link utilization, but the multi objective function proposed by the author not optimal in other aspects like response time and packet loss. A distortion factor (Taher and Besharat, 2008) for heterogeneous streams in traffic engineering of MPLS backbone networks in the presence of tunnelling and capacity constraints by formulating a distortion-aware non-linear discrete optimization problem. The author presented a two-phase heuristic approach to solve this formulation efficiently.

MPLS hierarchical architecture (Saljooghi and Rayeni, 2011; Mohamed et al., 2008) for label-switched networks can be used to address all required functions of converged/unified networks, from initial IP level authentication and configuration, security, session control, resource reservation, admission control, to quality of service and policy management, enhanced only where necessary to address the idiosyncrasies of the mobile wireless environment. This architecture encompassing mostly Internet Engineering Task Force (IETF) standardized protocols, takes advantage of MPLS flexibility to address wireless-specific requirements such as micro mobility as well as nonwireless specific requirements, such as traffic engineering and quality of service and does not involve 
specific requirements in the mobile terminal for initiating label-switched paths over the wireless interface and allowing end to end interconnection to the backbone network.

The performance of a Traffic Engineering (TE) strategy for MPLS based network (Lep and Mesarec, 2008), described in (Ab-Rahman et al., 2009) is carried out. Specifically the implementation based on a distributed control plane (Internet-like) has been investigated and realized by means of a test bed where Real Signaling Protocol (RSVP-TE) and routing protocols (OSPF-TE) have been implemented. All these previous works are provided feasibility but optimal result based on the current requirement of the internet users such as lower response time and lesser packet loss.

\section{MATERIALS AND METHODS}

This chapter describes the importance of traffic based routing and the related works on traffic based routing. With shortest path algorithms, the drawback of these algorithms is that when an arc is good for many source to destination pairs, some source-destination pairs will choose this arc for their path and can induce the collision on this arc. Traffic based routing algorithm does not only optimize network resource for present time, but also for future demands. Traffic based routing algorithms will predict the links that will be blocked if the route has traffics through them and will reduce routing traffic going through these links.

This problem can be described by math formulation: Set $\theta_{\text {sd }}$ is max flow of ingress-egress $(s, d)$ pair that is computed after satisfying setup Link State Packet (LSP) demand, solution of problem is maximize sum of $\theta_{\text {sd }}$ of all ingress-egress pairs. The optimization goal is Eq. 1:

$$
\max i m i s e \sum \theta_{\mathrm{sd}}
$$

The traffic on each link of each ingress-egress pair, setup the path with bandwidth D and satisfy constraints: total bandwidth of all traffic going through each link is less than residual bandwidth of this link and total traffic going in is equal traffic going out each node on networks. Solving complete problem is NP problem.

The (Lozano et al., 2008; Kerh et al., 2005) propose a mathematical programming model for the design of two-layer networks where MPLS is considered on top of transport networks (SDH or WDM depending on required link speed). This model take into account the tradeoff between the cost of adding MPLS support in the core nodes and the savings in the link bandwidth allocation due to the statistical multiplexing and the traffic grooming effects induced by MPLS nodes. The traffic matrix specifies for each point-topoint request a pair of values: A mean traffic value and an additional one. Using this traffic model, the effect of statistical multiplexing on a link allows allocating a capacity equal to the sum of all the mean values of the traffic demands routed on the link and only the highest additional one. The authors propose a path-based Mixed Integer Programming (MIP) model for the problem of optimizing the number (Basi and Murthy, 2004; Geem et al., 2005) and location of MPLS nodes in the network and the link capacities. They applied Lagrangian relaxation (Al-Mutairi et al., 2009; Moghimi, 2007) to this model and use the sub-gradient method to obtain a lower bound of the network cost. As the number of path variables used to model the routing grows exponentially with the graph size, the authors uses an initially limited number of variables and a column generation approach. Form the results it is observed that the proposed model will suit only for small size and real-world instances.

There are two main reasons for why TCP does not scale to high speed networks. First, packet loss is a binary congestion signal that conveys no information about the degree of congestion. Second, due to stability reasons, relying only on packet loss for congestion indication requires TCP to use a conservative window increment policy and an aggressive window decrement policy. In high speed networks, every loss event forces a TCP flow to perform a Multiplicative Decrease (MD), followed by the slow convergence of the Additive Increase (AI) algorithm to reach high utilization. Since the time for each individual AIMD epoch is proportional to the per-flow packets, TCP flows remain in low utilization regions for prolonged periods of time thereby resulting in poor link utilization. Using AQM/ECN in conjunction with TCP does not solve this problem since the (one-bit) ECN feedback, similar to packet loss, is not indicative of the degree of congestion either. XCP addresses this problem by precisely measuring the fair share of a flow at a router and providing explicit rate feedback to end-hosts. One noteworthy aspect of XCP is the decoupling of efficiency control and fairness control at each router.

XCP uses MIMD to control the flow aggregate and to converge exponentially fast to any available bandwidth and uses AIMD to fairly allocate the bandwidth among competing flows. As a consequence, $\mathrm{XCP}$ requires multiple bits in the packet header to carry bandwidth allocation information from network routers to end-hosts and congestion window and Round-Trip Time (RTT) information from the end-hosts to the network routers. 
There are few works proposed by (Mohan et al., 2008; Chandra and Baskaran, 2011) reached good result in wired and wireless environment. The proposed work, applies a traffic flow analysis methodology for identifying current traffic flow and based on the traffic, the proposed model desires the routing. The proposed wok is explained in the following algorithms.

\section{Proposed traffic flow based routing:}

Step 1: For every time interval, $(\Delta t)$, the MPLS periodically propagates hello packets to every other switch connected directly and indirectly in the sub net.

Step 2: Each MPLS switch calculates the load factor based on the data flow using the following Eq. 2. Where load factor is the term used to classifies the traffic pattern. In this study, the load factor is classified as three models, which are low traffic, medium traffic and high traffic.

$\mathrm{LFi}=\mathrm{ARi}+(\mathrm{kq} * \mathrm{QLi})) /\left(\mathrm{LCi}^{*} \Delta \mathrm{ti} * \mathrm{TUi}\right)$

where, LF represents the load factor of MPLS switch ' $i$ ', AR represents the arrival rate of the concern MPLS switch ' $\mathrm{i}$ ', kq is the constant, QL represents the Queue Length of the concern MPLS switch ' $i$ ', LC represents the Link Capacity of the connection established from the concern MPLS switch ' $\mathrm{i}$ ' and the TU represents the Target Utility of concern MPLS switch 'i'

Step 3:= Based on the Load factor, the traffic pattern is classified. If the LF factor of the MPLS switch ' $i$ ' is less than 0.8 then this may be labeled as low traffic. The medium traffic is termed as load factor higher than 0.8 and less than 1 and the high traffic is termed as load factor higher than 1.

Step 4 : Routing decision is taken based on the load factor of the paths. The path having low traffic is the highly priority route. And the medium traffic path is low priority route. The high traffic path is always avoided in order to avoid traffic.

The above algorithm may be applied as creamy layer on the top of any existing routing algorithm.

\section{RESULTS}

The performance analysis of proposed traffic analysis based routing model is given in the Table 1-6 and Fig. 2-4. The Table 1-3 shows the packet loss in various exiting routing protocols and the proposed routing protocols under normal, medium and heavy load condition.

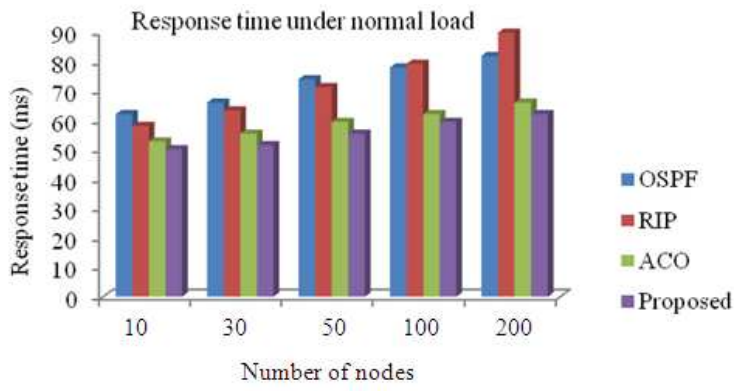

Fig. 2: Response time under normal load (in ms)

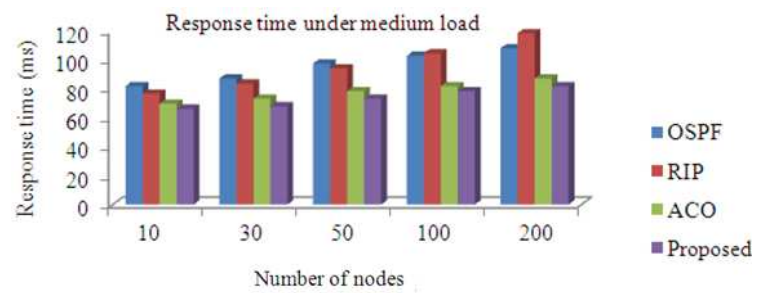

Fig. 3: Response time under medium load (in ms)

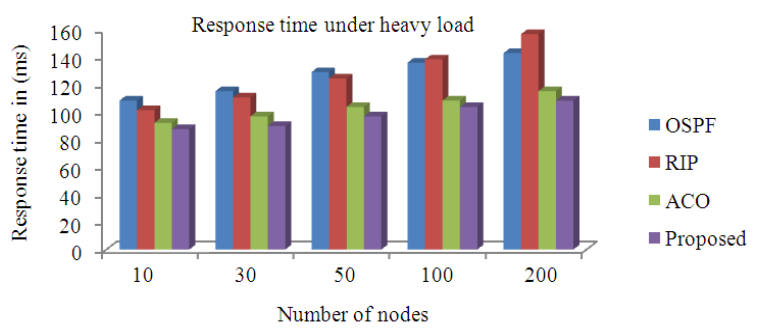

Fig. 4: Response time under heavy load (in ms)

Table 1: Packet loss in normal load (number of packets)

\begin{tabular}{lllll}
\hline Nodes & OSPF & RIP & ACO & Proposed \\
\hline 10 & 1 & 1 & 0 & 0 \\
30 & 3 & 1 & 0 & 0 \\
50 & 5 & 2 & 2 & 0 \\
100 & 5 & 19 & 3 & 0 \\
200 & 8 & 22 & 4 & 1 \\
\hline
\end{tabular}

Table 2: Packet loss in medium load

\begin{tabular}{lccll}
\hline Nodes & OSPF & RIP & ACO & Proposed \\
\hline 10 & 2 & 1 & 1 & 0 \\
30 & 2 & 1 & 1 & 0 \\
50 & 5 & 4 & 3 & 1 \\
100 & 8 & 35 & 5 & 1 \\
200 & 12 & 40 & 8 & 1 \\
\hline
\end{tabular}

Table 3: Packet loss in heavy load (number of packets)

\begin{tabular}{lcccl}
\hline Nodes & OSPF & RIP & ACO & Proposed \\
\hline 10 & 3 & 2 & 1 & 1 \\
30 & 3 & 2 & 1 & 1 \\
50 & 4 & 6 & 1 & 3 \\
100 & 14 & 63 & 12 & 4 \\
200 & 44 & 72 & 22 & 7 \\
\hline
\end{tabular}


Table 4: Response time in normal load (in ms)

\begin{tabular}{lllll}
\hline Nodes & OSPF & RIP & ACO & Proposed \\
\hline 10 & 62 & 58 & 53 & 50 \\
30 & 66 & 63 & 55 & 52 \\
50 & 74 & 71 & 59 & 55 \\
100 & 78 & 79 & 62 & 59 \\
200 & 82 & 90 & 66 & 62 \\
\hline
\end{tabular}

Table 5: Response time in medium load (in $\mathrm{ms}$ )

\begin{tabular}{lccll}
\hline Nodes & OSPF & RIP & ACO & Proposed \\
\hline 10 & 82 & 77 & 70 & 66 \\
30 & 87 & 84 & 73 & 68 \\
50 & 98 & 94 & 79 & 73 \\
100 & 103 & 105 & 82 & 79 \\
200 & 108 & 119 & 87 & 82 \\
\hline
\end{tabular}

Table 6: Response time in heavy load (in ms)

\begin{tabular}{lllcc}
\hline Nodes & OSPF & RIP & ACO & Proposed \\
\hline 10 & 108 & 101 & 92 & 88 \\
30 & 115 & 111 & 97 & 90 \\
50 & 129 & 124 & 104 & 97 \\
100 & 136 & 138 & 108 & 104 \\
200 & 143 & 157 & 115 & 108 \\
\hline
\end{tabular}

The Table 4-6 represents the average response time in various exiting routing protocols and the proposed routing protocols under normal, medium and heavy load condition. The Fig. 2-4 shows the average response time under normal, medium and heavy load conditions.

\section{DISCUSSION AND CONCLUSION}

From the above results, the proposed Traffic flow Analysis model provides better result than existing method. This traffic flow analysis model shows appreciate difference in the response time as well as packet loss. For the MPLS network, the proposed model suit well based on all network metrics.

\section{REFERENCES}

Ab-Rahman, M.S., S.A.C. Aziz and K. Jumari, 2009. Protection for an immediate split structure of treebased EPON architecture-ideal condition analysis. Am. J. Eng. Applied Sci., 2: 372-380. DOI: 10.3844/ajeassp.2009.372.380

Al-Mutairi, N., F. Al-Rukaibi and P. Koushki, 2009. Measurements and model calibration of urban traffic noise pollution. Am. J. Environ. Sci., 5: 613617. DOI: 10.3844/ajessp.2009.613.617

Basi, H.M.A. and M.B.R. Murthy, 2004. A simple scheme for improved performance of fixed outage rate cellular system. Am. J. Applied Sci., 1: 190192. DOI: 10.3844/ajassp.2004.190.192

Chandra, M.B. and R. Baskaran, 2011. Survey on recent research and implementation of ant colony optimization in various engineering applications. Int. J. Comput. Intell. Syst., 4: 566-582.

Geem, Z.W., K.S. Lee and Y. Park, 2005. Application of harmony search to vehicle routing. Am. J. Applied Sci., 2: 1552-1557. DOI: 10.3844/ajassp.2005.1552.1557

Kakooza, R., L.S. Laboobi and J.Y.T. Mugisha, 2005. Modeling traffic flow and management at unsignalized, signalized and roundabout road intersections. J. Math. Stat., 1: 194-202, DOI: 10.3844/jmssp.2005.194.202

Kerh, T., Y.M. Wang and Y. Lin, 2005. Experimental evaluation of anti-stripping additives mixing in road surface pavement materials. Am. J. Applied Sci., 2: 1427-1433. DOI: 10.3844/ajassp.2005.1427.1433

Lep, M. and B. Mesarec, 2008. Road transport congestion costs calculations-adaptation to engineering approach. Am. J. Applied Sci., 5: 2933. DOI: 10.3844/ajassp.2008.29.33

Lozano, A., F. Granados and J.P. Antun, 2008. Estimation of vehicle flows and emissions for various scenarios of street network modifications in Mexico City. Am. J. Environ. Sci., 4: 198-203. DOI: 10.3844/ajessp.2008.198.203

Moghimi, E., 2007. Necessary geomorphologic information for roads safety, with emphasize soleghan road in west of Tehran, Iran. Am. J. Environ. Sci., 3: 199-204. DOI: 10.3844/ajessp.2007.199.204

Mohamed, H., M.H. Lee, M. Sarahintu, S. Salleh and B. Sanugi, 2008. The use of taguchi method to determine factors affecting the performance of destination sequence distance vector routing protocol in mobile ad hoc networks. J. Math. Stat., 4: 194-198. DOI: 10.3844/jmssp.2008.194.198

Mohan, B.C., R. Sandeep and D. Sridharan, 2008. A data mining approach for predicting reliable path for congestion free routing using self-motivated neural network. Studies Comput. Intell., 149: 237246. DOI: 10.1007/978-3-540-70560-4_20

Saljooghi, F.H. and M.M. Rayeni, 2011. Distinguishing congestion and technical inefficiency in presence undesirable output. Am. J. Applied Sci., 8: 903-909. DOI: 10.3844/ajassp.2011.903.909

Taher, S.A. and H. Besharat, 2008. Transmission congestion management by determining optimal location of FACTS devices in deregulated power systems. Am. J. Applied Sci., 5: 242-247. DOI: 10.3844/ajassp.2008.242.247 